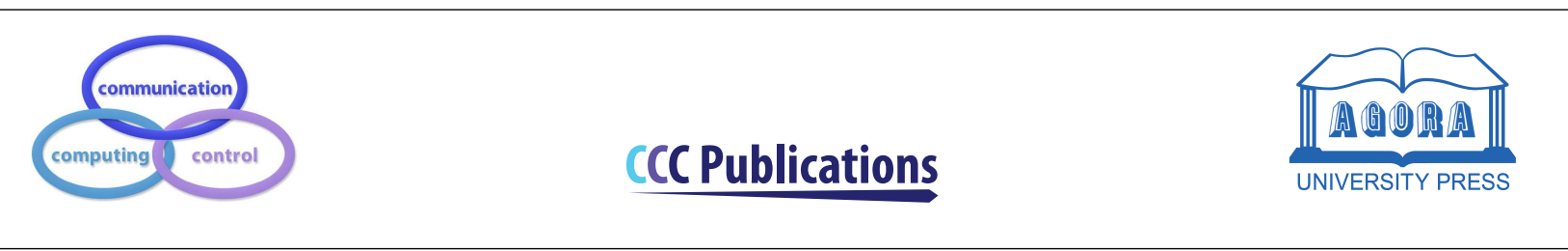

\title{
A Green Routing Mathematical Model for IoT Networks in Critical Energy Environments
}

\author{
C. Lozano-Garzon, G. A. Montoya, Y. Donoso
}

\section{Carlos Lozano-Garzon*}

Systems and Computing Engineering Department

Universidad de Los Andes

Bogotá, Colombia

*Corresponding author: calozanog@uniandes.edu.co

\section{German Adolfo Montoya}

Systems and Computing Engineering Department

Universidad de Los Andes

Bogotá, Colombia

ga.montoya44@uniandes.edu.co

\section{Yezid Donoso}

Systems and Computing Engineering Department

Universidad de Los Andes

Bogotá, Colombia

ydonoso@uniandes.edu.co

\begin{abstract}
In this paper, we propose a multi-objective mathematical optimization model that is the underlying support for the proposal of a new routing algorithm that aims to extend the lifetime in IoT networks for applications in critical energy environment. The network lifetime is evaluated for three approaches: the Hop Count approach, the Energy Consumption approach, and the Multiobjective approach based on Free Space Loss and the battery energy level of the IoT nodes. After this evaluation, we compared the different approaches in terms of how many transmissions were possible to do under a particular approach until none path cannot be found from an origin node to a destination node. Finally, we conclude that the Multi-objective method was the best strategy for extending the network lifetime since building short distance paths and considering battery level of the IoT nodes every time is, in the long run, a better strategy than just building paths considering nodes with a high battery level or building paths minimizing the number of network hops.
\end{abstract}

Keywords: Mathematical Optimization Model, Green Routing Algorithm, Internet of Things. 


\section{Introduction}

In recent years, the number of devices connected to the Internet has experimented an unforeseen growth due to mainly their easiness of use and large amounts of applications. However, one subclass of these devices has had one of the most significant growth: the IoT devices [3, 11, 12]. Nonetheless, it is necessary to propose improved techniques to manage the large amounts of data traffic generated by this type of network. In this sense, it is required to improve the usage of these resources; since otherwise, this will have repercussions in the amount of energy needed to keep the devices in a normal operation mode for sending and receiving information [10].

The solution that has been used to keep the sensors working is to improve the batteries attached to the devices $[2,10]$. However, lately, a technique has been used to lessen the energy shortage on these devices with no access to an electric network; this is Energy Harvesting [8]. Basically, it is a way to gather energy from the environment using hardware to transform this energy into electricity and then give it to the device. Some examples of energy that can be gathered are solar, thermal, piezoelectric, among others [4].

In this research, we focus our work on IoT networks that are deployed on locations without access to traditional electric energy supplies such as mountains, deserts, lakes, among others. These networks are usually used to gather information about the natural conditions of the environment where they are placed, such as temperature, humidity, acidity, and others that eventually lead scientists to know the state of that specific region [4]. Another characteristic of this type of network is that due to the lack of energy, not all IoT devices can use high energy consumption protocols for transmitting/receiving data and must rely on low energy consumption protocols such as ZigBee or Bluetooth LE [5].

However, current solutions are focused on either optimize the routing of data through the network or increasing the amount of energy available for the devices $[2,6,9]$. In this sense, we propose a new approach that not only takes into account the energy consumption in the batteries of each device, but also takes into account the amount of energy that it can harvest from the environment to find optimal paths in the IoT networks in order to extend as much as possible the network lifetime.

We propose a multi-objective mathematical optimization model; in this model we used of three objective functions, all of them for minimizing: the first one, based on the number of path hops; the second one, related to the energy consumption in the network; and the third one, based on the Free Space Loss indicator and the battery energy level of the nodes. Regarding the model constraints, these restrictions are designed to represent the fact of finding a minimum cost path to send an activated service from a source node to a base station considering flow conservation, bandwidth, and path building constraints for activated services. As a result our multi-objective proposal presents the best results in contrast with the shortest path or the energy consumption optimization strategies.

The remainder of this paper is organized as follows. The general problem statement is described in section 2; the mathematical optimization model for the problem and the routing algorithm are introduced in section 3 ; in section 4 we presents the results obtained; and finally, section 5 shows the conclusions and the future works.

\section{General problem statement}

In this work, we propose a mathematical optimization model and a routing algorithm for IoT networks according to the challenges involved in critical energy environment applications.

The main objective of our proposal is finding a minimum cost path to send an activated service from a source node to any base station doing an efficient use of the available energy of the IoT devices, minimizing the path hops, and reducing the distance involved in the discovered path with the aim of extending as much as possible the network lifetime.

From this perspective, our problem will involve more than one objective function that sometimes could be in conflict. For example, the shortest path (in terms of the number of hops) between two nodes could have the highest energy consumption. For this reason, we use a multi-objective approach in order to find an optimal set of solutions that holds a trade-off between all proposed objective functions. 
We assume that the IoT network has symmetrical links between the different nodes; therefore, we could model it as a non-directed graph. We represent the input graph as a set compound of a set of nodes and a set of links.

In the network and according to the example represented in the Figure 1, we could have several origin nodes $O$ (source nodes) and some destinations nodes $D$ (base stations). From an origin node, we could have several activated services $\left(S_{2}\right.$ for $O_{1}$ and $S_{1}$ for $\left.O_{2}\right)$ that must be sent to any base station. In real applications, a service is a specific type of information that must be transmitted from an origin node to a base station such as the temperature, the humidity or $\mathrm{pH}$ level. However, not all the origin nodes need to send all its services to a base station [7, 14]. For this reason, in this approach, we assume that each origin node could have activated and deactivated services to be sent to a base station. This network feature is approached in the mathematical optimization proposed in the next section.

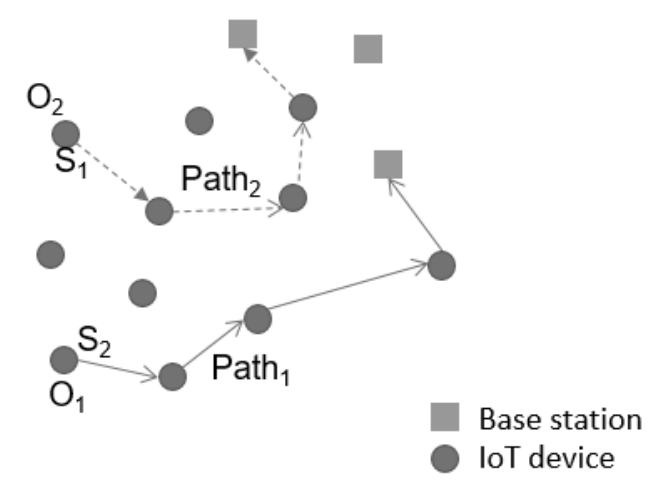

Figure 1: Example of an IoT network.

In summary, we have an IoT network with several origin nodes and destination nodes (base stations), at which is required to find an optimal path $\left(P a t h_{1}\right.$ and $\left.P a t h_{2}\right)$ to transmit an activated service from an origin node to a destination node (base station). This path can be built considering different goals, depending on what we want to achieve. In this sense, three goals are proposed to be obtained: minimizing the Hops Count, minimizing the Energy Consumption, and a Multi-objective approach that considers minimizing the energy consumption and the Free Space Loss Indicator. Once a goal is defined, we have to determine its performance in terms of network lifetime. Each of these goals and the method used to determine its network lifetime will be explained in the next sections.

\section{Mathematical optimization model approach}

In this section we present a multi-objective mixed-integer formulation of the mathematical model proposed for the problem described above.

\subsection{Notation}

The sets, parameters and variables required by our mathematical model are described in the Table 1.

\subsection{Objective functions}

Our mathematical optimization model is applied considering different objective functions, depending on what we want to optimize. In this sense, three objective functions are proposed to be optimized: the Hops Count, the Energy Consumption, and a Multi-objective approach that considers minimizing the energy consumption and the Free Space Loss Indicator. Each of these objective functions will be explained below. 
Table 1: Notations of the proposed model

\begin{tabular}{|c|c|}
\hline Sets & Description \\
\hline$N$ & Set of network nodes. \\
\hline$O$ & Set of origin nodes. \\
\hline$D$ & Set of destination nodes. \\
\hline$I$ & Set of intermediate nodes: $i \in N \mid i \notin O \cup D$ \\
\hline$S$ & Set of services. \\
\hline$O I$ & $O \cup I$ \\
\hline$I D$ & $I \cup D$ \\
\hline Parameters & Description \\
\hline Dist $_{i j}$ & Distance between an $i$-node and a $j-$ node. \\
\hline$u_{i j}$ & Bandwidth capacity for each $(i, j)$ link. \\
\hline$B^{s o}$ & Bandwidth required by a $s$-service from an $o$-origin node. \\
\hline$S_{a c t}^{s o}$ & Indicates which $s$-service is active to be sent from an $o$-origin node. \\
\hline$E c_{i}$ & Consumed energy at the $i$-node. \\
\hline Variables & Description \\
\hline$X_{i j}^{\text {sod }}$ & $\begin{array}{l}\text { Is a binary variable that determines if the link }(i, j) \text { is used to send a } s \text {-service from } \\
\text { an } o \text {-origin node to a } d \text {-destination node. }\end{array}$ \\
\hline$Y^{\text {sod }}$ & $\begin{array}{l}\text { Is a binary variable that determines if the } s \text {-service from the } o \text {-origin node will } \\
\text { arrive at the } d \text {-destination node. }\end{array}$ \\
\hline
\end{tabular}

\subsubsection{Hops count}

The hops count function is the main function of many routing algorithms. This function represents the number of links through which packets must pass from source to the set of destination nodes. The purpose of minimizing function 1 is finding the shortest path between any pair of nodes.

$$
\min \sum_{i \in N} \sum_{j \in N} \sum_{s \in S} \sum_{o \in O} \sum_{d \in D} X_{i j}^{s o d}
$$

\subsubsection{Energy consumption}

An IoT network could be composed of heterogeneous nodes, and each one of them could have different energy consumption in both transmission and reception. Given the nature of IoT networks, some nodes that belong to this network could be mobile phones, sensors, or laptops. Therefore, the proposed routing algorithm, in order to reduce power consumption, should select the path with the lower overall energy consumption to increase the battery life of these kind o devices. The objective function 2 takes into account both the energy consumption used by the source node in the transmission process and the energy used by the destination node in the reception process, over the entire path.

$$
\min \sum_{i \in N} \sum_{j \in N} \sum_{s \in S} \sum_{o \in O} \sum_{d \in D} E c_{i} * E c_{j} * X_{i j}^{s o d}
$$

For the energy consumption model is required to take into account additional considerations. If a node has to send a data packet of $K$ bits to another node located at a distance $D$, then, the following are the expressions to calculate the energy consumption in the transmitter node as well as the receiver node. In the transmitter node, the consumption is $E_{\text {elec }}+E_{a m p}$, where $E_{\text {elec }}$ is the energy consumption for codification, modulation and filtering. $E_{a m p}$ corresponds to energy consumption for the Transmitter Power Amplifier. In the same way, in the receiver node, the consumption corresponds to $E_{a m p}$. Then, the expressions for the transmitter and receiver sensor are the following:

$$
E_{t x}=\left(E_{\text {elec }}+E_{a m p}\right) * K * D^{2}
$$




$$
E_{r x}=E_{a m p} * K * D^{2}
$$

In the constraint 3 there will have a higher energy consumption than constraint 4 because for transmission is required an extra consumption for codification, modulation and filtering $\left(E_{\text {elec }}\right)$, in addition to energy consumption for amplifying the signal received $\left(E_{a m p}\right)$.

\subsubsection{Free space loss}

This function defines the signal strength loss in free space conditions. This loss is directly related with the distance between the nodes and the transmission frequency. The aim of the following objective function 5 is the minimization of the maximum overall loss in the path.

$$
\min \sum_{i \in N} \sum_{j \in N} \sum_{s \in S} \sum_{o \in O} \sum_{d \in D} D_{i s t_{i j}} * X_{i j}^{s o d}
$$

\subsubsection{Multi-objective approach (Energy consumption + Free space loss)}

This function combines our previous energy consumption function plus a function based on the Free Space Loss indicator, which represents the signal strength loss in free space conditions and is directly related with the distance between the nodes and the transmission frequency. In this sense, the Free Space Loss indicator (FSL) will be proportional to the distance between two pairs of nodes. The FSL indicator between two nodes is given by the following expression [1] [15]:

$$
F S L_{i j}(d B)=20 \log _{10} D_{i j}+20 \log _{10} f+92.45
$$

Where $D_{i j}$ corresponds to the distance $(\mathrm{km})$ between two pair of nodes and $f$ is an operational frequency used in a IoT technology to perform transmissions and receptions measured in GHz. Once an IoT technology is determined, its operational frequency is considered fixed for doing FSL calculations. However, while the operational frequency and the term 92.45 are considered constants in the FSL expression, the distance $D_{i j}$ impacts proportionally the FSL calculation since $D_{i j}$ is a parameter that changes depending on the distance between two pair of nodes.

The multi-objective function 7 is described in the following equation.

$$
\begin{gathered}
\min \sum_{i \in N} \sum_{j \in N} \sum_{s \in S} \sum_{o \in O} \sum_{d \in D}\left(w_{1} * E c_{i} * E c_{j} * X_{i j}^{s o d}+w_{2} * F S L_{i j} * X_{i j}^{s o d}\right) \\
\text { where } w_{1}+w_{2}=1
\end{gathered}
$$

\subsection{Model constraints}

According to the general problem statement, some activated services must be sent from origin nodes to any destination node (base station). This scenario is denoted in the following constraints:

\subsubsection{Origin Nodes}

The expressions 8 and 9 allow us selecting only one destination per activated service. An activated service represents a service that is required to be sent from an origin node to any destination node; whether a service is activated or not is determined by the parameter $S_{a c t}^{s o}$.

$$
\begin{gathered}
\sum_{d \in D} Y^{s o d}=1 * S_{a c t}^{s o} \quad \forall s \in S, \forall o \in O \\
\sum_{j \in N \mid j \in I D} X_{i j}^{s o d}=1 * Y^{s o d} \quad \forall i \in N, \forall o \in O \mid i \in O \quad \forall s \in S, \forall d \in D
\end{gathered}
$$




\subsubsection{Destination nodes}

Expressions 10 and 11 allow just one destination per service. If $Y^{\text {sod }}$ is activated, that is, it is necessary to send a service $s$ from $o$ to $d$, we guarantee that one destination $d$ is selected to receive the service $s$.

$$
\begin{gathered}
\sum_{i \in N \mid i \in O I} X_{i j}^{s o d}=1 * Y^{s o d} \quad \forall j \in N, \forall d \in D \mid j \in D \quad \forall s \in S, \forall o \in O \\
\sum_{i \in N \mid i \in D} X_{i j}^{s o d}=0 \quad \forall j \in N, \forall d \in D \mid j \in D \quad \forall s \in S, \forall o \in O
\end{gathered}
$$

\subsubsection{Intermediate nodes}

Expression 12 represents the flow conservation law in order to find a path between $s$ and $d$ for an activated service $s$.

$$
\sum_{j \in N \mid j \in I D} X_{i j}^{s o d}-\sum_{j \in N \mid j \in O I} X_{j i}^{s o d}=0 \quad \forall i \in I, \forall s \in S, \forall o \in O, \forall d \in D
$$

\subsubsection{Bandwidth}

Expression 13 assures that different services could be transmitted in a specific link (i,j). Otherwise, the services must be sent for different $(i, j)$ links.

$$
\sum_{s \in S} \sum_{o \in O} \sum_{d \in D} B^{s o} * X_{i j}^{s o d} \leq u_{i j} \quad \forall i \in N, \forall j \in N
$$

\subsubsection{Undirected graph}

Due to we are dealing with undirected graphs (a link between $i$ and $j$ is equivalent to have a link $(\mathrm{i}, \mathrm{j})$ and a link $(\mathrm{j}, \mathrm{i}))$ expression 14 guarantees that, if between nodes $i$ and $j$ a link must be selected, just one of them is selected.

$$
X_{i j}^{s o d}+X_{j i}^{s o d} \leq 1 \quad \forall i \in N, \forall j \in N, \forall s \in S, \forall o \in O, \forall d \in D
$$

In summary, we have mathematical optimization model formulation that represents an IoT network with several origin nodes and destination nodes (base stations), at which is required to find optimal paths to transmit activated services from origin nodes to destination nodes (base stations). Depending on which objective function is considered, these paths are built minimizing the hops count, considering an efficient usage of the available energy of the IoT devices, or considering a third approach which is a result of taking into account the energy consumption of IoT nodes plus minimizing the Free Space Loss indicator. Therefore, our next step consists of proposing a method to determine the network lifetime for each approach, which will be explained in the next section.

\subsection{Routing algorithm using the mathematical model solution}

The previous mathematical model allows sending particular services from different origin nodes to any destination node for one time. The term "one time" must be understood as the mathematical model is executed one time to obtain a solution. However, due to network purpose corresponds to send as many services as possible until nodes energy allows it, the mathematical model must be performed several times in order to know the network lifetime. The algorithm 1 illustrates how the mathematical model is handled to obtain the network lifetime. 
Our goal consists of finding a path to carry an activated service from an origin node to a destination node in a network; however, if our scenario is a disconnected network, it is possible that this path cannot be found. This means we have obtained an infeasible solution. For this reason, the mathematical model must run considering a connected network in order to find feasible solutions, which is guaranteed in the first line. The network randomness is in terms of nodes coordinates, guaranteeing that each node is not disconnected from the network.

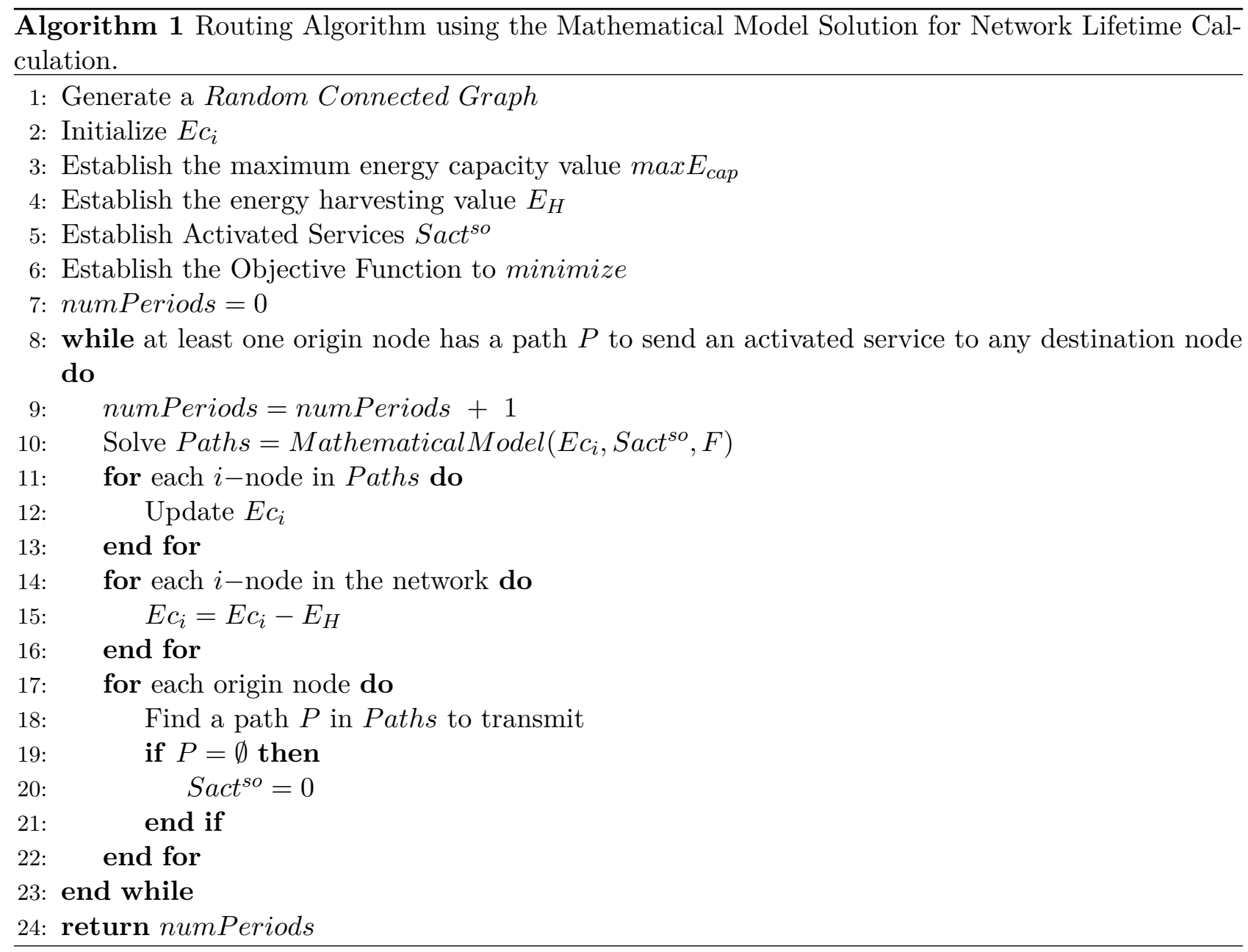

Line 2 shows the consumed energy of each node, which is initialized with a default value of 1 . As nodes are selected for transmitting or receiving activated services, they increase their consumed energy.

Line 3 establishes the maximum energy capacity of each node. This value is the same for all nodes, except for destination nodes. Destination nodes are a particular type of nodes in IoT; usually, they are devices with enhanced processing, memory and battery resources in comparison with the rest of network devices. For this reason, we assume that this type of nodes has no limitations in terms of energy consumption. Concerning the rest of the network nodes, if the consumed energy of an $i$-node, $E c_{i}$, surpasses the maximum energy capacity, $\max E_{c a p}$, this node has wasted all of its battery energy capacity, and then, this node is not available anymore for transmitting or receiving a service in the network.

Line 4 defines the energy harvesting value. This value is decreased from the $E c_{i}$ due to it is an energy amount coming from a harvesting technology such as solar panels.

Line 5 establishes which services will be activated; that is, from all available services in the origin nodes, which of them finally require to be sent from an origin node to a destination node. In other words, by modifying $S a c t^{\text {so }}$ ( 1 or 0 ), we can define which service will be activated or not. From line 8 to 24 , a Period means how many times it is possible to do transmission from an origin node to a destination node. Each time that it is possible to find at least one path from an origin node to a destination node, numPeriods is increased, that is, the network lifetime has been extended. If none 
path is found, it means that some nodes required for building any path from an origin node to a destination node have depleted all of its available energy.

In the Implementation and Results sections, numPeriods is equal to the number of transmissions, that is, the number of times at which it was possible to send services from all source nodes to a destination node before all these source nodes have depleted all of its available energy.

\section{Implementation}

Our mathematical optimization model was implemented using GAMS and MATLAB in order to show the performance of each approach, that is, Hops Count, Energy consumption, and the Multiobjective approaches. Our implementation scenarios were divided into two parts: the first one, called "Basic Scenario", implements an illustrative scenario in order to understand the basis of each result approach, and the second one, called "Complex Scenarios", implements many scenarios in order to obtain statistical results.

\subsection{Basic scenario}

We established a simple scenario to determine if our mathematical model was correctly defined. Figure 2 shows the defined testing scenario, where each link value indicates the Free Space Loss (FSL) value. Besides the Free Space Loss value of each link in Figure 2, on Table 2 we summarize others important parameters assumed in the network scenario.

According to the Table 2, additional details will be specified:

- The number of network nodes is 7, where two nodes (1 and 2) are source nodes, and the destination nodes are nodes 6 and 7 . In other words, an activated service from a particular source node needs to be sent to any of the destination nodes.

- The activated services for the source node 1 is just the service 1 , and the activated service for the source node 2 is just the service 2 . That is, source node one does not have the second service activated and it is not necessary to send this service to a destination node. In the same way, source node two does not have the first service activated.

- We assume all nodes begin with $99 \%$ full of battery, except node 4 which starts with $90 \%$ of battery. We assume these values in order to establish whether the different approaches are building the paths correctly.

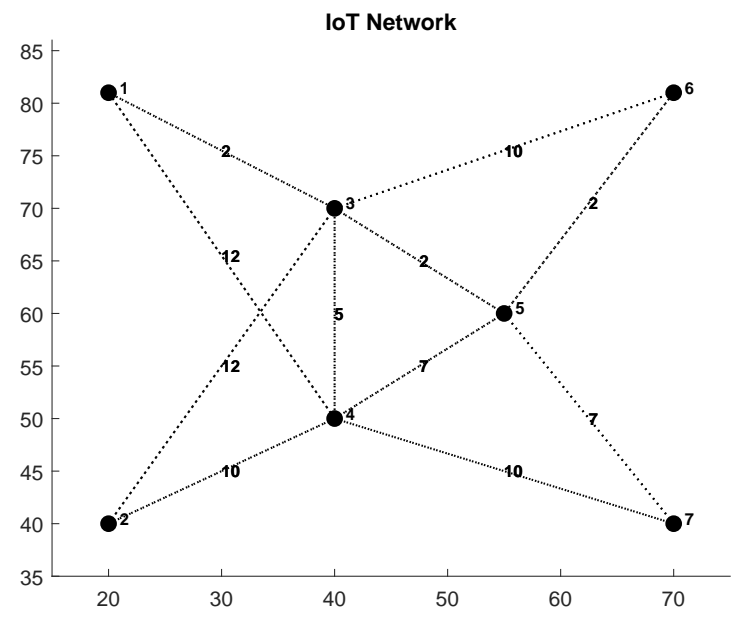

Figure 2: IoT network testing scenario. 
Based on Figure 2 and Table 2, we obtain the performance of our mathematical model for each approach, that is, the Hops Count approach, the Energy Consumption approach and the Multiobjective approach.

Table 2: Basic Scenario parameters.

\begin{tabular}{ll}
\hline Parameters & Value \\
\hline Work Area & $100 \times 100\left[\mathrm{~m}^{2}\right]$ \\
Eamp & $100\left[\mathrm{pJ} / \mathrm{bit} / \mathrm{m}^{2}\right]$ \\
Eelec & $50[\mathrm{~nJ} / \mathrm{bit}]$ \\
Number of IoT nodes & 7 \\
Sources nodes & 1 and 2 \\
Destination nodes (Gateways) & 6 and 7 \\
Number of services & 2 \\
Activated services & $S_{1}$ at $1, S_{2}$ at 2 \\
$E_{c_{i}} \forall i \in N \mid i \neq 4$ & $1 \%$ of total battery \\
$E_{c_{4}}$ & $10 \%$ of total battery \\
\hline
\end{tabular}

\subsection{Complex scenarios}

In this section, we enunciate the details of the scenarios evaluated in order to obtain statistical results. In other words, each approach is performed many times at different network sizes in order to obtain statistical results. Figure 2 show the typical networks considered to be evaluated by each approach.

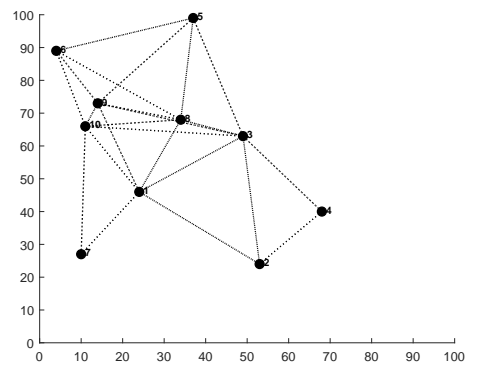

(a) Typical network of 10 nodes.

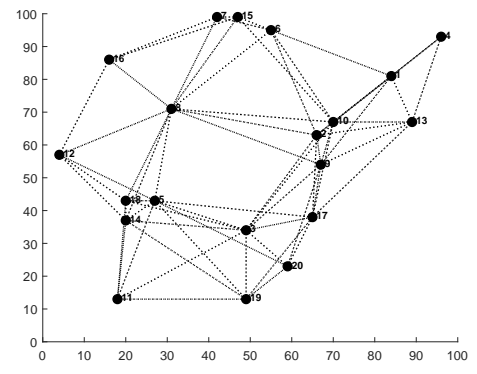

(b) Typical network of 20 nodes.

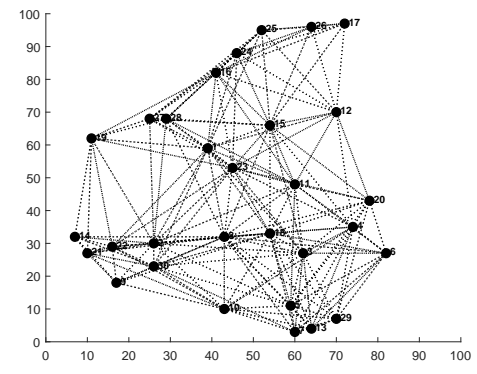

(c) Typical network of 30 nodes.

Figure 3: Typical networks sizes evaluated.

According to the Table 3, additional details will be specified:

Table 3: Complex Scenarios parameters.

\begin{tabular}{ll}
\hline Parameters & Value \\
\hline Work Area & $100 \mathrm{x} 100\left[\mathrm{~m}^{2}\right]$ \\
Eamp & $100\left[\mathrm{pJ} / \mathrm{bit} / \mathrm{m}^{2}\right]$ \\
Eelec & $50[\mathrm{~nJ} / \mathrm{bit}]$ \\
Number of IoT nodes & $10,15,20,25$, and 30 \\
Sources nodes & Depending on the scenario: one source node, \\
& two source nodes or three source nodes \\
Destination nodes (Gateways) & The last node \\
Number of services & 1 \\
Activated services & One service for each source node \\
$E_{c_{i}} \forall i \in N$ & $1 \%$ of total battery \\
Runs for each approach & 100 \\
\hline
\end{tabular}


- The goal is to evaluate the approaches considering different network sizes, that is, a network with 10, 15, 20, 25, 30 and 35 nodes. With this rule we aim to analyze how each approach reacts to different network sizes. At this point, we want to remark that with more than 40 nodes, the mathematical optimization model found a solution after a pair of hours.

- Each approach was evaluated considering a different number of source nodes; specifically when the network had one source node (the node 1), two source nodes (the nodes 1 and 2) or three source nodes (the nodes 1,2 and 3). Also, for simplicity, we only considered one activated service for each source node. Finally, the destination node always was the last node of the network; e.g. in a network with 20 nodes, the node 20 was the destination node (the gateway).

- We assume all nodes begin with an energy consumption of $1 \%$; in order to guarantee that all nodes start with the same energy conditions.

- We assume 100 runs for each approach at each network size. The total time for all runs was approximately more than one week.

\section{Results}

\subsection{Basic scenario}

The Basic Scenario results are divided into two parts. The first one is related with the solution path for each approach (Hops Count, Energy consumption, and the Multi-objective approach). This mean, that given the Table 2, we calculate and show the solution path for each approach in order to compare their results. The second part, of the basic scenario results, corresponds to the evaluation of the network lifetime for each approach; through this analysis we want to determine which method is better in terms of performing more transmissions in the network.

It is important to remark that, according to the Routing Algorithm using the Mathematical Model Solution Section, the number of transmissions is equal to numPeriods; that is the number of times at which it was possible to send all activated services from all source nodes to a destination node, before all these source nodes have depleted all of its available energy.

Here below, we are going to show and discuss the first part results.

Figures $4 \mathrm{a}$ and $4 \mathrm{~b}$ represent the Hops Count performance. Due to we are minimizing the number of hops, we obtain paths with the minimum possible number of hops regardless of using IoT nodes with low battery (node 4). Besides, this approach does not care using links with a high Free Space Loss value, which implies more energy consumption since more re-transmissions are needed to amend the signal attenuation.

Figures $4 \mathrm{c}$ and $4 \mathrm{~d}$ show the Energy Consumption performance. This approach considers the current battery levels of nodes with the intention of, in the long run, achieving extends the network's life. According to these figures, the solution paths avoid using node 4, since it has a low battery; however, this is a result for an specific instant. Thus, we have to verify how much, in the long term, this approach preserves the network lifetime.

Figures $4 \mathrm{e}$ and $4 \mathrm{f}$ indicate the Multi-objective approach performance. Due to we are minimizing the sum of Free Space Loss values of all links and taking into account the energy consumption in each IoT node. This approach tends to reduce the use of energy in terms of re-transmissions, for signal attenuation, and also reduce the use IoT nodes with a low battery level. As in the previous approach, we need to check how much, in the long term, this approach preserves the network lifetime.

According to the evaluation of the network lifetime for each approach, we assume the same parameters shown in Table 2 , but considering that $E_{c_{i}}=0 \% \forall i \in N$. Therefore, all IoT network nodes have full battery at the beginning of the model evaluation to guarantee a fair distribution of energy in all network nodes. The results of this evaluation is presented in the Table 4.

According to Table 4, the Hops Count approach is clearly the worst method to apply in an IoT network in terms of network lifetime since it allows only 632 transmissions, compared against 701 and 763, from the Energy Consumption and the Multi-objective approach, respectively. 


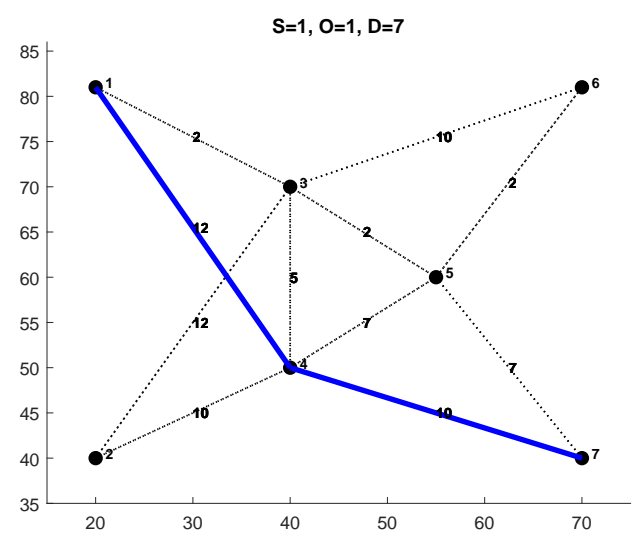

(a) Hops performance for the source node 1 .

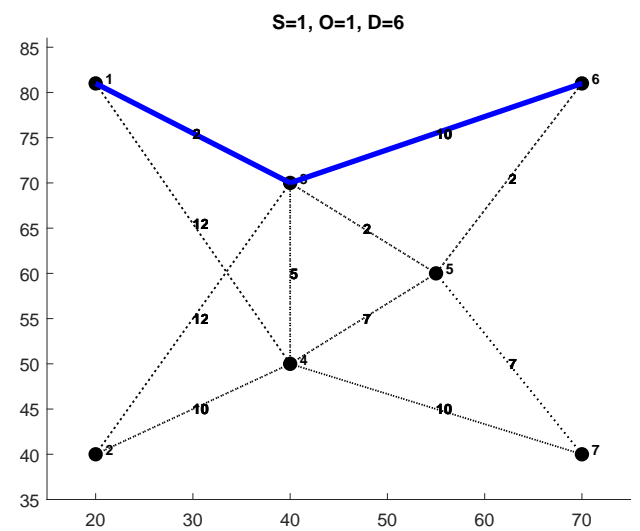

(c) Energy Consumption performance for the source node 1 .

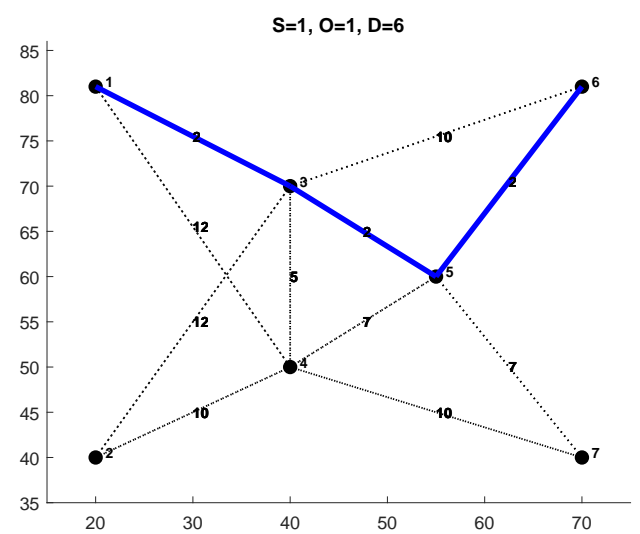

(e) Multi-objective performance for the source node 1.

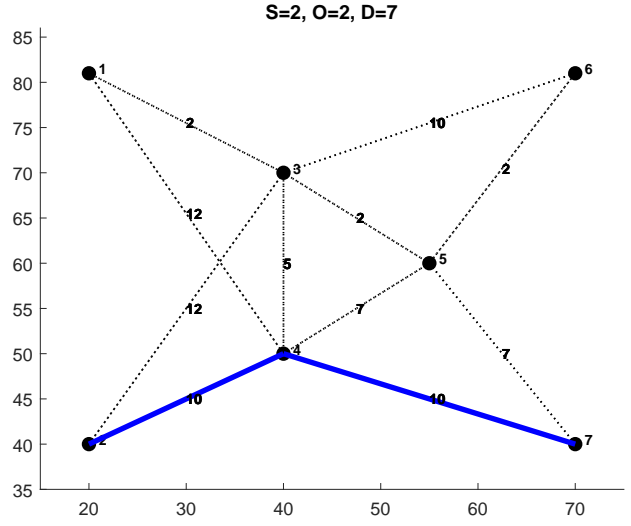

(b) Hops performance for the source node 2 .

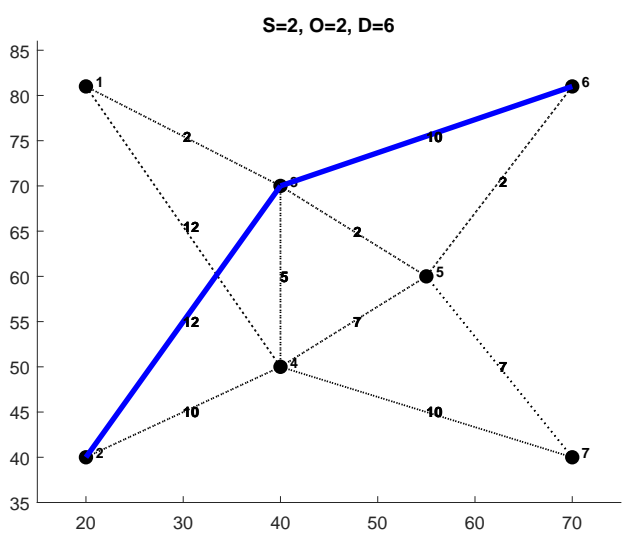

(d) Energy Consumption performance for the source node 2 .

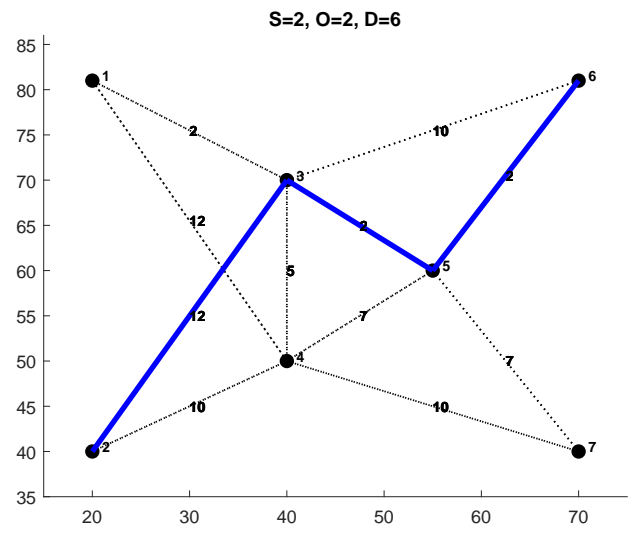

(f) Multi-objective performance for the source node 2 .

Figure 4: Performance for each approach.

Table 4: Network lifetime performance.

\begin{tabular}{lc}
\hline Approach & Number of Transmissions \\
\hline Hops & 632 \\
Energy consumption & 701 \\
Multi-objective approach (Energy + FSL) & 763 \\
\hline
\end{tabular}


As we expected, the Energy Consumption method obtained the second place extending the network lifetime. Even though this method uses the network nodes with less energy consumption (with a high battery level), in the long term, this strategy is not finally the best one due to it does not take into account the impact of using links with a high Free Space Loss indicator. This fact allows the network to waste more energy than expected.

Finally, the Multi-objective approach is the best method for extending the network lifetime since the signal strength has a significant impact on the network energy consumption, in addition to considering the battery level of the nodes (the Energy Consumption approach). In other words, building short distance paths every time and considering the battery level of the nodes is, in the long term, a better strategy than just building paths with a high battery level of the nodes.

\subsection{Complex scenarios}

Based on the parameters presented in Table 3, we obtain the results of each approach in terms of transmissions performed against the network size. As we mention above, the number of transmissions is equal to numPeriods; which is the number of times at which it was possible to send all activated services from all source nodes to a destination node before all these source nodes have depleted all of its available energy.

The first part of the results obtained are presented in Figure 5. The sub-figures shown the performance of each approach in terms of the number of transmissions as both the network size and the number of source nodes increase.

According to Figures 5a, 5b and $5 \mathrm{c}$ we can observe that, as the network size increases the number of transmissions also increases. In other words, as the number of network nodes increases, increases the probability that the direct neighbors of the source node are closer to it; allowing low distances from these nodes to the source. These low distance implies transmissions with low energy consumption, extending the network lifetime; and, hence, increasing the number of transmissions. This behavior applies to all approaches because, directly or indirectly, all of them depend on the distance.

In addition, for each network size the best results was obtained by the Multi-objective approach; while the worst was the Hops count approach. This behavior is coherent according to the results shown in the Basic Scenario results. This mean that the network size does not affect the results of the studied approaches.

Likewise, in the figures $5 \mathrm{~b}$ and $5 \mathrm{c}$ it is observed that increasing the number of source nodes increases the number of transmissions. The fact of having two source nodes increases the number of transmissions compared against the one source node scenario presented in Figure 5a; and in the same way, the fact of having three source nodes increases the number of transmissions compared against the one and two source nodes scenarios shown in Figure 5a and Figure 5b.

It is possible that when one source node has consumed all of its energy, the other source nodes can still be alive. For this reason, the number of transmissions is increased.

Finally, all figures of this section (Complex Scenarios) show that all approaches present an increasing performance as the network size grows; this performance increasing exhibits a slight ascendant exponential behavior. This behavior can be explained by the fact that as the size of the network increases, the number of neighboring nodes increases for each node; and thus, exist more connections between them. This implies that there is more chance of finding other alternative paths to reach the gateway; and there is therefore a higher probability of increases the number of transmissions in the network.

Based on a complete graph, the increase in the number of connections is represented by $\frac{n(n-1)}{2}$ where $n$ is the number of nodes [13]. For example, when $n=2,3,4,5,6,7 \ldots$ the total connections are 1, 3, 6, 10, 15 and 21 respectively, which exhibits an exponential growth. Therefore, due to the number of connections increases exponentially, the path availability also grows in an exponential manner, represented by the increasing of the number of transmissions. 


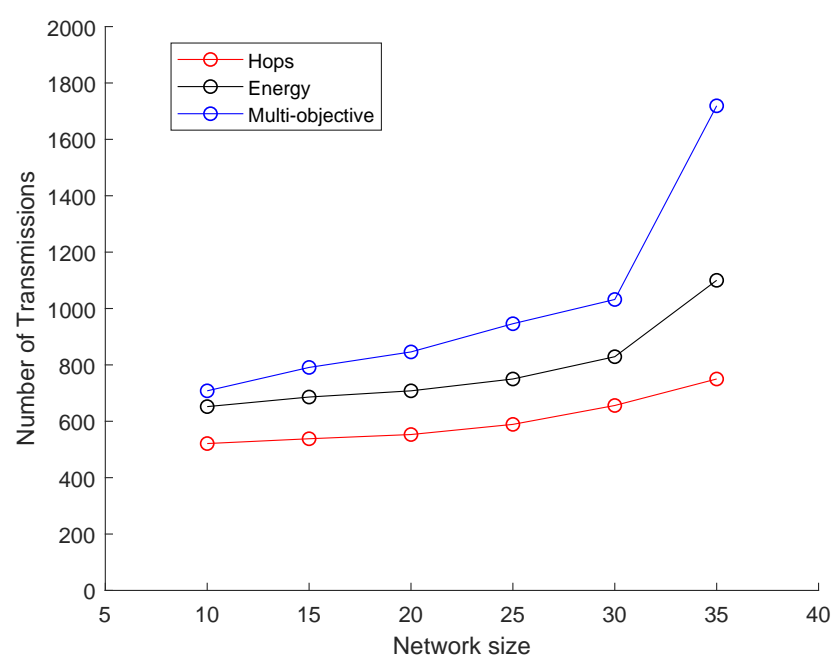

(a) Approaches performance for one source node.

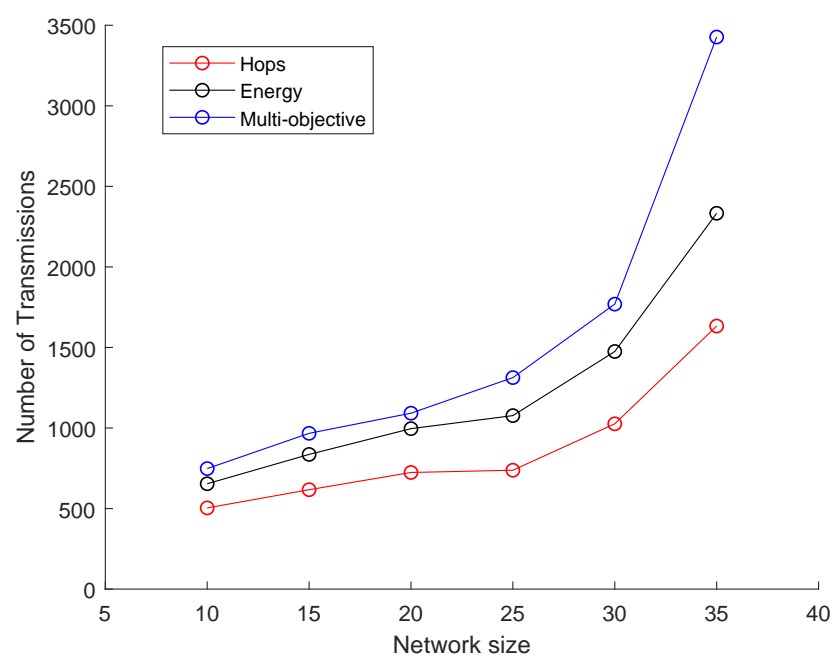

(b) Approaches performance for two source nodes.

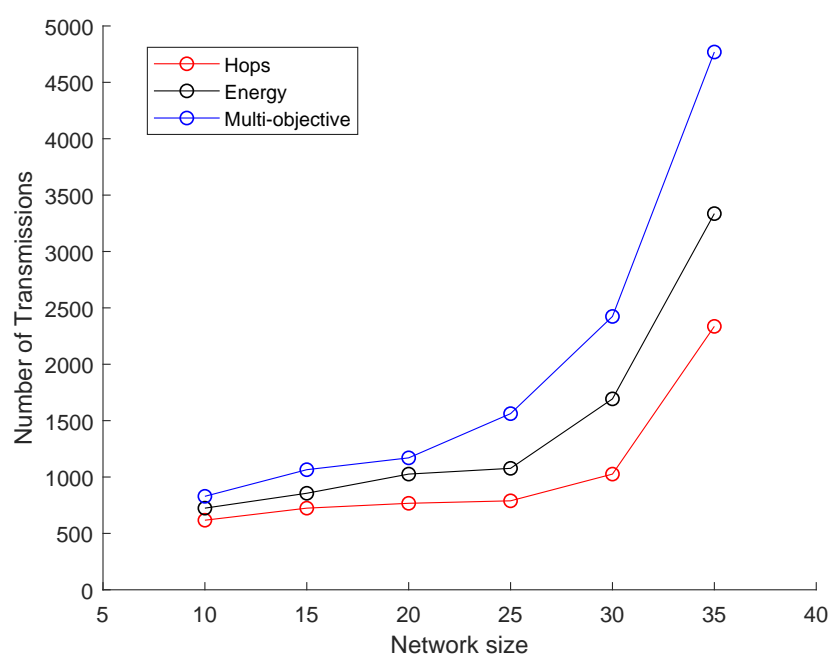

(c) Approaches performance for three source nodes.

Figure 5: Approaches performance against the network size. 


\section{Conclusion}

In this work we propose a multi-objective mathematical optimization model as the basis for a green routing algorithm; this algorithm aims to extend the lifetime in IoT networks for applications in critical energy environments. Our model considers the activating/deactivating of services from a specific source IoT node in order to reflect how IoT networks operate currently.

The routing algorithm proposed obtain the optimal lifetime network calculations for three different approaches: the Hop Count approach, the Energy Consumption approach, and the Multi-objective approach (Energy consumption + Free Space Loss). In this sense, the lifetime of each approach was evaluated in terms of the number of transmissions performed until were consumed the battery of the nodes.

As a result of our tests, the multi-objective approached obtained the best performance in the long term, this mean that building a short distance paths and considering the battery level of the IoT nodes every time is the best strategy for this kind of environments. The proposed model could be used to develop a new green routing protocol, and also, as a theoretic reference for new routing algorithms.

In addition, it is important to remark as a result, that independently of the used strategy, as the network size grows, its lifetime increases. For this reason, it is recommended to implement applications with the most large amount of nodes in order to increase the network lifetime.

For future works, we are planning to test real scenarios using physical IoT nodes in order to confirm the simulation results. For this purpose, we will use Raspberry Pi motes to implement the different approaches seen previously to evaluate the network lifetime and confirm which approach is the best strategy in real-world scenarios.

\section{Author contributions}

The authors contributed equally to this work.

\section{Conflict of interest}

The authors declare no conflict of interest.

\section{References}

[1] Akyildiz, I.F.; Vuran, M.C. (2010). Wireless Sensor Networks, John Wiley \& Sons Ltd, 2010.

[2] Alvi, S. A.; Shah, G. A.; Mahmood, W. (2015). Energy efficient green routing protocol for Internet of Multimedia Things, 2015 IEEE Tenth International Conference on Intelligent Sensors, Sensor Networks and Information Processing (ISSNIP), Singapore, 1-6, 2015.

[3] Cho, Y; Kim, M; Woo, S. (2018). Energy Efficient IoT based on Wireless Sensor Networks for Healthcare, 20th International Conference on Advanced Communication Technology (ICACT, Chuncheon-si Gangwon-do, Korea (South), 294-299, 2018.

[4] Dong, Y.; Wang, J.; Shim, B.; Kim, D.I. (2016). DEARER: A Distance-and-Energy-Aware Routing With Energy Reservation for Energy Harvesting Wireless Sensor Networks, IEEE Journal on Selected Areas in Communications, 34(12), 3798-3813, 2016.

[5] Elbassiouny, S.O.; Hassan, A.M. (2015). Energy-efficient routing technique for Wireless sensor Networks under energy constraints, 2015 International Wireless Communications and Mobile Computing Conference (IWCMC), Dubrovnik, 647-652, 2015.

[6] Farhan, L.; Kharel, R.; Kaiwartya, O.; Quiroz-Castellanos, M.; Raza, U.; Teay, S.H. (2018). LQOR: Link Quality-Oriented Route Selection on Internet of Things Networks for Green Computing, 2018 11th International Symposium on Communication Systems, Networks \& Digital Signal Processing (CSNDSP), Budapest, 1-6, 2018. 
[7] Hasan, M.Z.; Al-Turjman, F.; Al-Rizzo, H. (2018). Analysis of Cross-Layer Design of Qualityof-Service Forward Geographic Wireless Sensor Network Routing Strategies in Green Internet of Things, IEEE Access, 6, 20371-20389, 2018.

[8] Hu, J.; Luo, J.; Zheng, Y.; Li, K. (2019). Graphene-Grid Deployment in Energy Harvesting Cooperative Wireless Sensor Networks for Green IoT, IEEE Transactions on Industrial Informatics, 15(3), 1820-1829, 2019.

[9] Kumar, N.; Vidyarthi, D.P. (2018). A Green Routing Algorithm for IoT-Enabled Software Defined Wireless Sensor Network,IEEE Sensors Journal, 18(22), 9449-9460, 2018.

[10] Liu, X; Ansari, N. (2018). Dual-Battery Enabled Green Proximal M2M Communications in LPWA for IoT, 2018 IEEE International Conference on Communications (ICC), Kansas City, MO, 1-6, 2018 .

[11] Noje, D.; Tarca, R.; Dzitac, I.; Pop, N. (2019). IoT Devices Signals Processing based on Multidimensional Shepard Local Approximation Operators in Riesz MV-algebras, International Journal of Computers Communications \& Control, 14(1), 56-62, 2019.

[12] Noje, D.; Dzitac, I.; Pop, N.; Tarca, R.(2020). IoT Devices Signals Processing Based on Shepard Local Approximation Operators Defined in Riesz MV-Algebras, Informatica, 31(1), 131-142, 2020.

[13] Voloshin, V. (2009). Introduction to Graph Theory. Nova Science Publishers, Inc. 2009.

[14] Wang, D.; Wang, X.; Liang, Y.; Wang, Z. (2017). A Service Oriented Routing Scheme for Internet of Things, 2017 IEEE International Conference on Internet of Things (iThings) and IEEE Green Computing and Communications (GreenCom) and IEEE Cyber, Physical and Social Computing (CPSCom) and IEEE Smart Data (SmartData), Exeter, 683-688, 2017.

[15] Zheng, J.; Jamalipour, A. (2009) Wireless Sensor Networks: A Networking Perspective, John Wiley \& Sons Ltd, 2009.

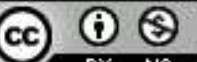

Copyright (C) 2020 by the authors. Licensee Agora University, Oradea, Romania.

This is an open access article distributed under the terms and conditions of the Creative Commons Attribution-NonCommercial 4.0 International License.

Journal's webpage: http://univagora.ro/jour/index.php/ijccc/

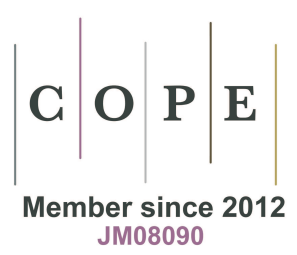

This journal is a member of, and subscribes to the principles of,

the Committee on Publication Ethics (COPE).

https://publicationethics.org/members/international-journal-computers-communications-and-control

Cite this paper as:

Lozano-Garzon, C.; Montoya, G. A.; Donoso, Y. (2020). A Green Routing Mathematical Model for IoT Networks in Critical Energy Environments, International Journal of Computers Communications \& Control, 15(4), 3914, 2020. https://doi.org/10.15837/ijccc.2020.4.3914 